\title{
Cafe Au Lait Spot
}

National Cancer Institute

\section{Source}

National Cancer Institute. Cafe Au Lait Spot. NCI Thesaurus. Code C40460.

A light brown, sharply demarcated skin patch. It is a manifestation of neurofibromatosis type 1 and McCune-Albright syndrome. 